\title{
Circular RNA expression profiles and features in NAFLD mice: a study using RNA-seq data
}

\author{
Xinlu Yuan ${ }^{1}$, Jianjun Diao ${ }^{2}$, Anqing Du ${ }^{3}$, Song Wen ${ }^{1}$, Ligang Zhou ${ }^{1 *+}$ and Yangbin $\operatorname{Pan}^{4^{*}+}$ (]
}

\begin{abstract}
Background: Nonalcoholic fatty liver disease (NAFLD) is primarily characterized by the hepatic cholesterol accumulation. Circular RNA (circRNA), one of noncoding RNA, involves in many liver diseases progression. However, no recent studies on circRNA expression profiles in NAFLD have been reported previously.

Methods: A NAFLD mouse model was constructed by providing high-fat diet (HFD) for 32 weeks. The circRNAs expression profile in normal mice and NAFLD mice were determined using high-output RNA sequencing method and bioinformatics methods, while the differentially expressed circRNAs were confirmed using Sanger sequencing and qRT-PCR. The circRNA-miRNA network was also predicted. The biological functions of circRNAs were annotated by Gene Ontology (GO) and Kyoto Encyclopedia of Genes and Genomes (KEGG).

Results: The results demonstrated the successful construction of NAFLD mice model by immunohistology and serology assay. In total, 93 dysregulated circRNAs were observed, including 57 upregulated circRNAs and 36 downregulated circRNAs, in the NAFLD group. The circRNA-miRNA network revealed the complex interaction between circRNAs and its potential miRNA targets in NAFLD. The characteristic of tissue-specific expression in circRNA was demonstrated. The differentially expressed circRNAs with important biological function were also annotated using GO and KEGG. Both DDAH1 and VAV3 genes were found to be associated with the NAFLD development.
\end{abstract}

Conclusions: Taken together, this study demonstrated the circRNAs expression profile and features in NAFLD, which may provide potential biological markers for the pathogenesis of NAFLD.

Keywords: Nonalcoholic fatty liver disease; circular RNA, Expression profile, Tissue specificity, Regulatory network

\section{Background}

Nonalcoholic fatty liver disease (NAFLD) represents a common chronic liver disease in many developing and developed countries [1]. The prevalence of NAFLD is $20-40 \%$ in adult, while approximately $70-80 \%$ of occurrence in diabetic and obesity patients. NAFLD

\footnotetext{
*Correspondence: zhouligang@yahoo.com; endosubmit@126.com

${ }^{\dagger}$ Ligang Zhou and Yangbin Pan contributed equally to this study

1 Department of Endocrinology and Metabolic Diseases, Shanghai

Pudong Hospital, Fudan University Pudong Medical Center, 2800

Gongwei Road, Pudong, Shanghai 201399, China

${ }^{4}$ Department of Nephrology, Shanghai Pudong Hospital, Fudan

University Pudong Medical Center, 2800 Gongwei Road, Pudong, Shanghai 201399, China

Full list of author information is available at the end of the article
}

encompasses a series of clinical manifestations, such as simple hepatic steatosis and nonalcoholic steatohepatitis (NASH). NASH, an important stage in NAFLD, can even develop to fibrosis, cirrhosis and hepatocellular carcinoma (HCC) [2]. Recently, the occurrence of NAFLD has increased at an alarming rate with the rapid growth of obesity in the population. There is growing evidence that NAFLD can be caused by multiple factors, including lipid accumulation in liver, mitochondrial dysfunction, a high fat diet, insulin resistance and genetic factors [3]. Although the underlying mechanism of NAFLD has not been completely explained, recent researches have suggested to find out potential biomarkers that might be able to do early prediction and diagnosis for patients with NAFLD.

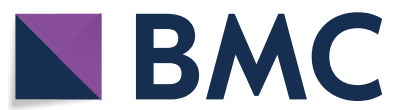

c) The Author(s) 2020. This article is licensed under a Creative Commons Attribution 4.0 International License, which permits use, sharing, adaptation, distribution and reproduction in any medium or format, as long as you give appropriate credit to the original author(s) and the source, provide a link to the Creative Commons licence, and indicate if changes were made. The images or other third party material in this article are included in the article's Creative Commons licence, unless indicated otherwise in a credit line to the material. If material is not included in the article's Creative Commons licence and your intended use is not permitted by statutory regulation or exceeds the permitted use, you will need to obtain permission directly from the copyright holder. To view a copy of this licence, visit http://creativeco mmons.org/licenses/by/4.0/. The Creative Commons Public Domain Dedication waiver (http://creativecommons.org/publicdomain/ zero/1.0/) applies to the data made available in this article, unless otherwise stated in a credit line to the data. 
Non-coding RNA molecules consist of transfer RNA (tRNA), microRNA (miRNA), and circular RNA (circRNA), which contributed greatly to many biological processes [4]. CircRNA was firstly discovered in 1976 in the viruses, which is a closed continuous loop with the deficiency of $5^{\prime}$ and $3^{\prime}$ end, in other words, circRNA cannot be degraded by RNase $\mathrm{R}$ and present with high stability in cells [5]. In addition, circRNA can also inhibit the miRNAs function which was found to be related to the progression and pathogenesis of chronic liver diseases [6]. The expression of circRNA is identified as extremely low in cells, however, the rapid advance of high-throughput sequencing methods has led to the high expression level of this molecule [7]. Numerous studies have revealed that the majority of circRNAs can interact with miRNA to regulate the target gene expression, that is, circRNA may be a potential biomarker and therapeutic target of NAFLD $[8,9]$. Therefore, the scientific community's awareness of circRNAs has been raised due to its unique characteristics. Recently, the circRNAs expression profiles have been reported to mediate neurological diseases, diabetes and lung metastasis. A previous study conducted by Qu et al. [10], have suggested a small number of dysregulated circRNAs in the NASH mice fed with methionine/choline deficient diet, and constructed a complex circRNA-miRNA pathway interaction. However, at this stage, not much information on the circRNAs expression profiles in hepatic tissues of NAFLD have been reported.

This study demonstrated the differentially expressed circRNAs in an NAFLD mouse model. This study aimed to find out the potential roles of circRNAs in the progression and pathogenesis of NAFLD. The RNA sequencing, RT-qPCR validation and multiple bioinformatics technologies were used to investigate the differentially expressed circRNAs, followed by identify a circRNAmiRNA interaction network that is closely associated with the progression of NAFLD.

\section{Materials and methods}

\section{Establishment of NAFLD mice model}

Totally 20 male mice (8 weeks old, weighting 16-20 g) were obtained from Changzhou Cavens Experimental Animal Co., Ltd. All animals were kept in plastic cages at a constant temperature of $25{ }^{\circ} \mathrm{C}$, humidity of $40 \%$ and $12 \mathrm{~h}$ light $/ 12 \mathrm{~h}$ dark cycle. The mice also had free food and water access during the experiment. In addition, we randomly divided the mice into two groups and provided with different diets for 32 weeks: control group fed with standard chow $(\mathrm{n}=10)$ and NAFLD group fed with highfat diet (HFD) $(n=10)$. The HFD was purchased from the Research Diets, Inc., New Brunswick, NJ (60\% kcal fat; D12492). The levels of blood glucose were measured after fasting for $12 \mathrm{~h}$. After that, all mice were anesthetized and sacrificed to collect blood for biochemical analysis, while the livers were harvested and weighted immediately. Serum and liver samples were stored in liquid nitrogen at $-80{ }^{\circ} \mathrm{C}$ for subsequent experiments. A small pieces of hepatic tissues were fixed in $4 \%$ paraformaldehyde (PFA) $>48 \mathrm{~h}$ at $4{ }^{\circ} \mathrm{C}$ for histological analysis. This study was approved by the Animal Research Ethical Committee of the Fudan University Pudong Medical Center. All experimental procedures followed the Guidelines for the Care and Use of Laboratory Animals of the National Institute of Health in China.

\section{Biochemical analysis}

Glucose tolerance test (GTT) and insulin tolerance test (ITT) were determined after fasting for $12 \mathrm{~h}$. The $1.5 \mathrm{~g} \mathrm{~kg}^{-1}$ glucose was injected intraperitoneally to conduct GTT. In contrast, $0.5 \mathrm{IU} \mathrm{kg}^{-1}$ insulin was injected for ITT. After injection, the blood glucose level was determined using OneTouch Ultra Glucose Test Strips (LifeScan Inc., Milpitas, CA) at different timelines, including 0, 30, 60, 90, and $120 \mathrm{~min}$.

Plasma triglyceride (TG) was measured using Triglyceride Kit (Wako Diagnostics, Richmond, VA), while the Cholesterol Assay Kit (BioVision, Irvine, CA) was used to determine the level of total cholesterol (TC). Plasma insulin was also measured with a MILLIPLEX $\left({ }^{\circledR}\right)$ MAP Mouse Metabolic Magnetic Bead Panel kit following the manufacturer's instructions. To determine the hepatic TG and TC levels in control and NAFLD group, hepatic tissues were rinsed with phosphatebuffered saline (PBS) and collected in isopropanol. The homogenate was generated after centrifugation at $12000 \times g$ for $15 \mathrm{~min}$ and then incubated at $4{ }^{\circ} \mathrm{C}$. The supernatants were used for further analyses.

\section{Histological analysis}

The hepatic tissues from each mouse were fixed in $4 \%$ paraformaldehyde, followed by dehydrated in grades of alcohol, and embedded in paraffin wax (Sakura, Tokyo, Japan). The sections with $5 \mu \mathrm{m}$ thickness were stained with hematoxylin and eosin (H\&E). The slices were also stained with Oil Red O (ORO) to analyze the accumulation of hepatic lipid. A light microscope was used to observe histological features of liver tissues under $\times 200$ (Olympus, Tokyo, Japan).

\section{RNA isolation and quality control}

Total RNA was extracted and purified using TRIzol reagent (Invitrogen, CA, USA) and RNeasy Mini kit (Qiagen). The RNA concentration was assessed using a spectrophotometer (NanoDrop ND-1000, Thermo 
Scientific), while the RNA integrity was measured using electrophoresis.

\section{The analysis of CircRNA sequencing}

RNA sequencing was applied using RNA samples from each group, while the RNase $R$ was treated to remove linear RNAs from isolated RNA. The amplified circRNAs were reverse transcribed into cDNA using random primers following the manufacturer's instructions. The cDNA was then synthesized and purified using the QiaQuick PCR extraction kit (Qiagen). The cDNA library was finally prepared according to illumine TruSeq library preparation instruction. CircRNA sequencing was conducted on an Illumina HiSeqTM 4000 (Illumina, CA, U.S.A).

\section{Reverse transcription-quantitative polymerase chain-reaction (RT-qPCR)}

RT-qPCR was carried out not only to validate the results of RNA sequencing, but also to detect the expression of DDAH1 and VAV3. The isolated RNAs from liver tissues were reversely transcribed into cDNA using the RT-PCR kits (Takara) in accordance with the manufacture's protocol, followed by amplifications using a SYBR Premix Ex Taq kit (Takara). The thermal conditions are as follows: $95^{\circ} \mathrm{C}$ for $5 \mathrm{~min}$, followed by 40 cycles of $10 \mathrm{~s}$ at $95^{\circ} \mathrm{C}, 60{ }^{\circ} \mathrm{C}$ for $30 \mathrm{~s}$ and $72{ }^{\circ} \mathrm{C}$ for $30 \mathrm{~s}$. $\beta$-actin was used as the internal reference. PCR bands were gel-purified and sequenced using Sanger method. The algorithm $2-\Delta \Delta C q$ method was applied to normalize the relative gene expressions to $\beta$-actin. The primer sequences used for RT-qPCR are listed in Table 1 and Additional file 4: Table S3.

\section{RNA sequencing and bioinformatics analysis}

The raw data with low-quality reads were filtered out using Qubit3.0, while the remaining reads were mapped to the mouse genome (GRCm38) using Bowtie2 v2.2.8. The reference genome was established by software
TopHat2 v2.1.1 $(25,26)$. The remaining reads after alignment were subjected to CIRCexplore, MapSplice and CircRNA_finder software for circRNA identification. The chromosome distribution of the identified circRNAs were evaluated. The circRNAs were categorized as significantly differentially expressed using edgeR package (fold change $\geq 1.5$ and $p$-value $<0.05$ ). The differentially expressed circRNAs were selected using volcano plotting. Gene ontology (GO) was applied to annotate meaningful gene products, which contains three categories of biological function, namely biological process (BP), cellular components (CC) and molecular function (MF). In contrast, Kyoto Encyclopedia of Genes and Genomes (KEGG) was utilized to identify the target genes in biological pathways.

Once the differentially expressed circRNAs are identified, StarBase v2.0 software was used to predict the target miRNAs. Mireap (https://sourceforge.net/proje cts/mireap/), Miranda v3.3a, (http://miranda.org.uk/) and TargetScan v7.0, (http://www.targetscan.org) databases were applied to predict the novel circRNAs. After that, the circRNA-miRNA network was visualized using Cytoscape software. To improve the reliability of our prediction, the match score was set as $>140$ and the minimum free energy $<-20$.

\section{Statistical analysis}

All data are expressed as mean \pm standard deviation. An independent sample t-test was carried out to determine differences between groups using SPSS (version 23.0; IBM Corp., Armonk, NY, USA). Statistical significance was set at $p<0.05$.

\section{Results}

The construction of the NAFLD mouse model

NAFLD mouse model was successfully constructed after 32 weeks HFD, as evidenced by significant fluctuations

Table 1 Primers sequences of selected circRNAs and internal reference for reverse transcription-quantitative polymerase chain-reaction (RT-qPCR) validation

\begin{tabular}{|c|c|c|c|}
\hline Forward Sequence $\left(5^{\prime}-3^{\prime}\right)$ & Name & Reverse Sequence $\left(5^{\prime}-3^{\prime}\right)$ & Length \\
\hline 5'-GCTTCTAGGCGGACTGTTAC-3' & $\beta$-actin-R1 & 5'-CCATGCCAATGTTGTCTCTT-3' & $100 \mathrm{bp}$ \\
\hline 5'-GCTCCTGGGAAGGTGACATC-3' & mmu_circ_0015959-R2 & 5'-CCTGAGGAGTTTCCTGGAAG-3' & $144 \mathrm{bp}$ \\
\hline 5'-TGTGGCCGATTCTTTGCATT-3' & mmu_circ_0010514-R2 & 5'-CATCATGTCAACCTTGAGGG-3' & $109 \mathrm{bp}$ \\
\hline 5'-CTTAGATCAGCCGTGTTGTG-3' & mmu_circ_0004580-R2 & 5'-ACAGTCCCATTAAGCCTTGC-3' & $144 \mathrm{bp}$ \\
\hline 5'-ATAGATGGCTGGGGCTTTGG-3' & chr7_82671604_82674582-R1 & 5'-GCGAACCGCTGTTGATACTT-3' & $100 \mathrm{bp}$ \\
\hline 5'-GCGACTCAGACACAGATCCA-3' & chr17_66049785_66053091-R1 & 5'-ACTGCTGTCACTGTCAGAAT-3' & $126 \mathrm{bp}$ \\
\hline 5'-AACACTCTGCACGGGTCAAG-3' & chr4_46734057_46749582-R1 & 5'-TCCGAAGCTGCTCTAGAATG-3' & $203 \mathrm{bp}$ \\
\hline 5'-GAACGTCCTATATCATTAGGG-3' & Chr11_94036599_94048558-R4 & 5'-TTCAAGTCTGCTGACTTCAG-3' & $161 \mathrm{bp}$ \\
\hline 5'-CATGAACTGCAGGGCTGAAC-3' & chr5_118593332_118593570-R1 & 5'-GCAGACAGCGGATGAAACTT-3' & $134 \mathrm{bp}$ \\
\hline
\end{tabular}




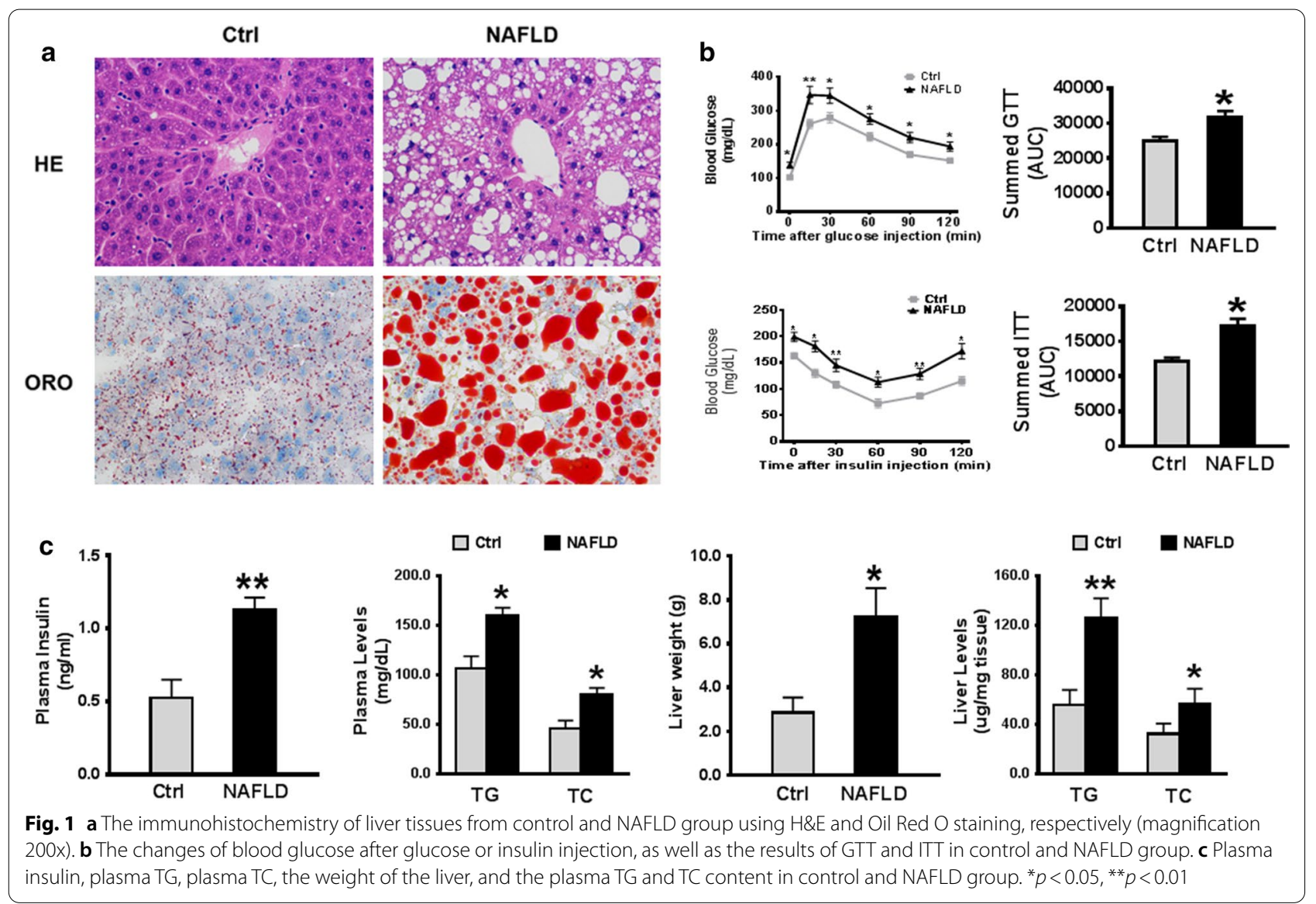

in serum levels and the presentation of hepatic tissues. The results of H\&E staining demonstrated the distinct histopathological features of NAFLD. As shown in Fig. 1, sections from NAFLD model group had disordered liver lobules, excessive fat-containing vacuoles in cytoplasm, and cellular swelling. The HFD-induced mice demonstrated the accumulation of fat in the liver under the ORO staining (Fig. 1a). Compared to controls, the blood glucose levels in the NAFLD group were significantly higher after glucose injection, especially at time period of $30 \mathrm{~min}$. Both NAFLD and control groups had declined blood glucose after insulin injection at different timeframe, but the level of blood glucose in NAFLD group was still higher than the control group (Fig. 1b). Both summed GTT and ITT of NAFLD group were significantly raised $(p<0.05)$. Additionally, the levels of plasma
TC, TG, plasma insulin and liver weight were dramatically elevated compared to the controls $(p<0.05)$. We also determined the levels of TG and TC within the livers. Our data reflected the hepatic TC and TC levels were noticeably elevated in the NAFLD group (Fig. 1c). These data in serum-based biomarkers and hepatic tissues indicated the successfully construction of the NAFLD mice model.

\section{Expression profile of circRNAs in NAFLD mice}

To identify the differentially expressed circRNAs between the NAFLD group and control group, circRNA sequencing was conducted in each group. A circular diagram represented the types and distributions of identified circRNAs on the chromosome (Fig. 2a). The length of lines

Fig. 2 Bioinformatics analysis of differentially expressed circRNA in NAFLD and normal mice. a The distribution of differentially expressed circRNA on mouse chromosome. The length of lines indicates the relative size of fold-change; Red: up-regulated circRNAs; Green: down-regulated circRNAs. b The distribution of circRNA length. Blue: not significantly expressed circRNAs; Orange: the significantly expressed circRNAs. c Heat maps reflected different circRNA expression profiles between NAFLD group and control group. $\mathbf{d}$ Volcano plots comparing differentially expressed circRNA in NAFLD mice with control. Red: up-regulated circRNAs; Green: down-regulated circRNAs. e The expression levels of eight random selected circRNAs after RT-qPCR analysis. ${ }^{*} p<0.05,{ }^{* *} p<0.01$ 


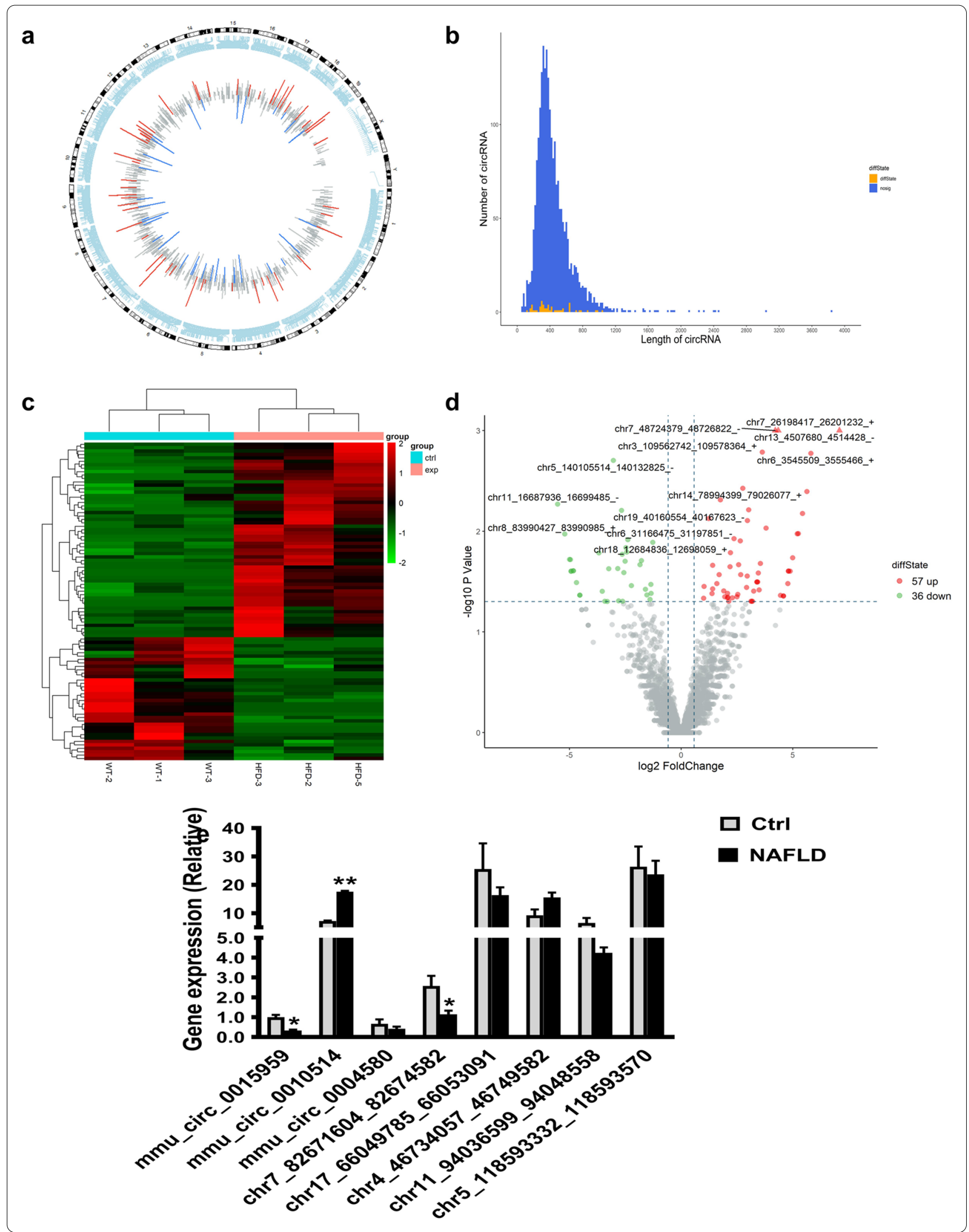




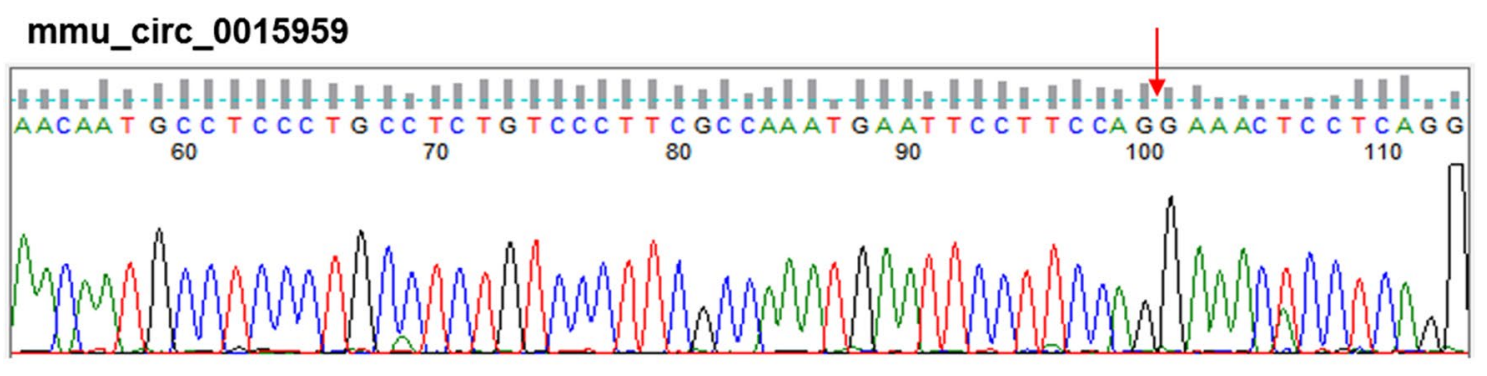

mmu_circ_0010514

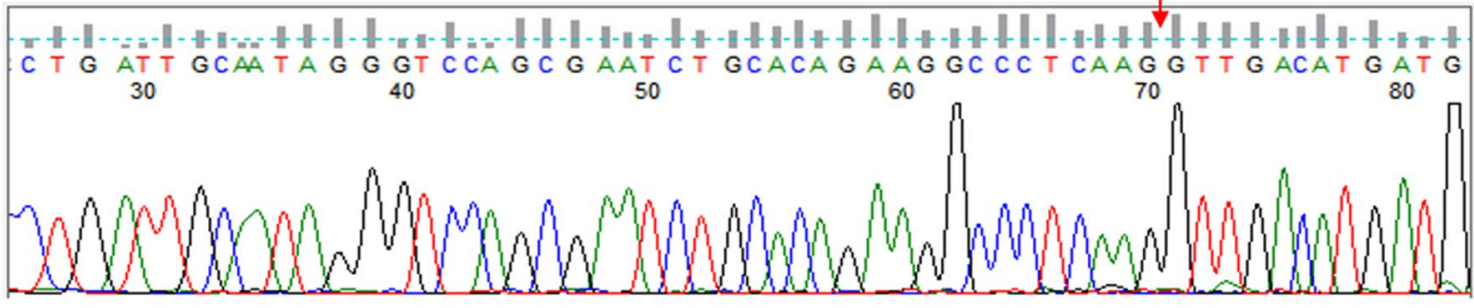

mmu_circ_0004580

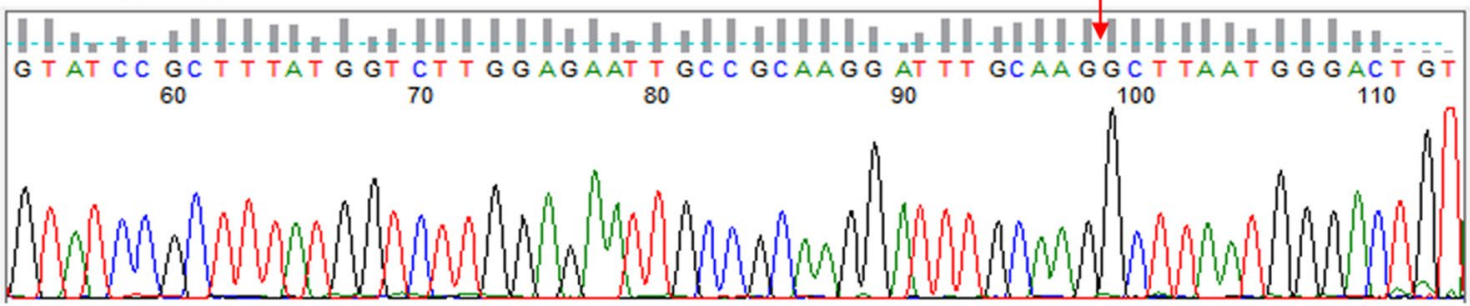

chr7_82671604_82674582

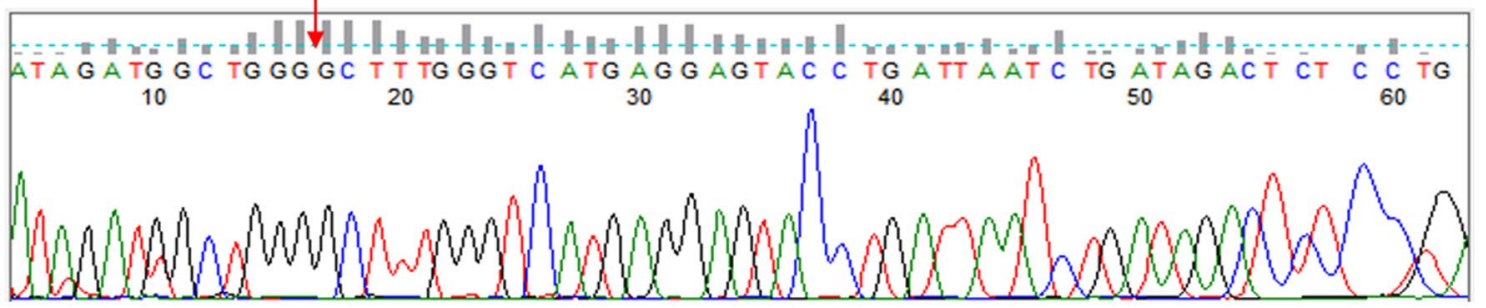

\section{chr17_66049785_66053091}

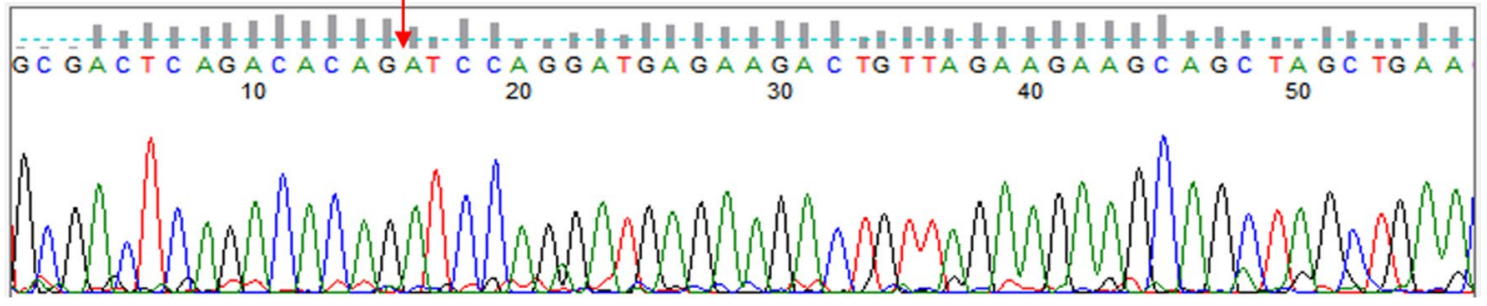

Fig. 3 Head-to-tail splicing in the RT-qPCR product of eight random selected circRNAs

indicates the fold-change. As shown in Fig. 2b, the distribution of circRNAs length was showed with the significantly differentially expressed circRNA in orange and in-differentially expressed circRNA in blue. In addition, hierarchical clustering and volcano plot exhibited the significant differentially expressed circRNAs in NAFLD and controls (Fig. 2c, d; FC $\geq 1.5, p<0.05$ ). Hence, 93 circRNAs of NAFLD mice were considered as dysregulated, in which 57 of upregulated circRNAs and 36 of downregulated circRNAs (Additional file 1: Table S1). Overall, 


\section{chr4_46734057_46749582}

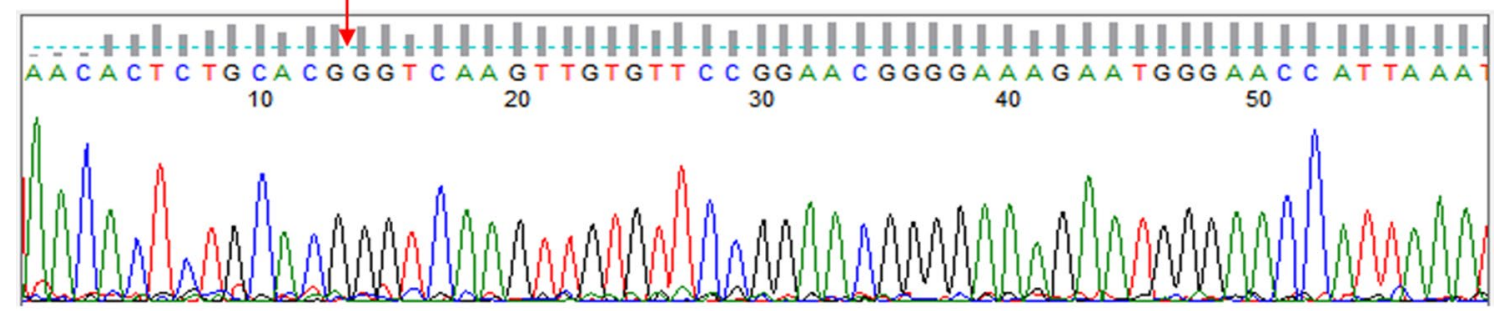

\section{chr11_94036599_94048558}

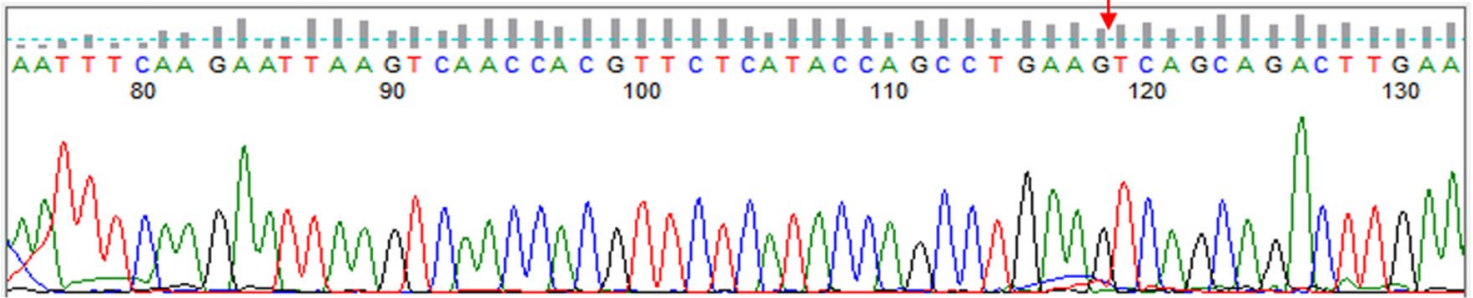

chr5_118593332_118593570

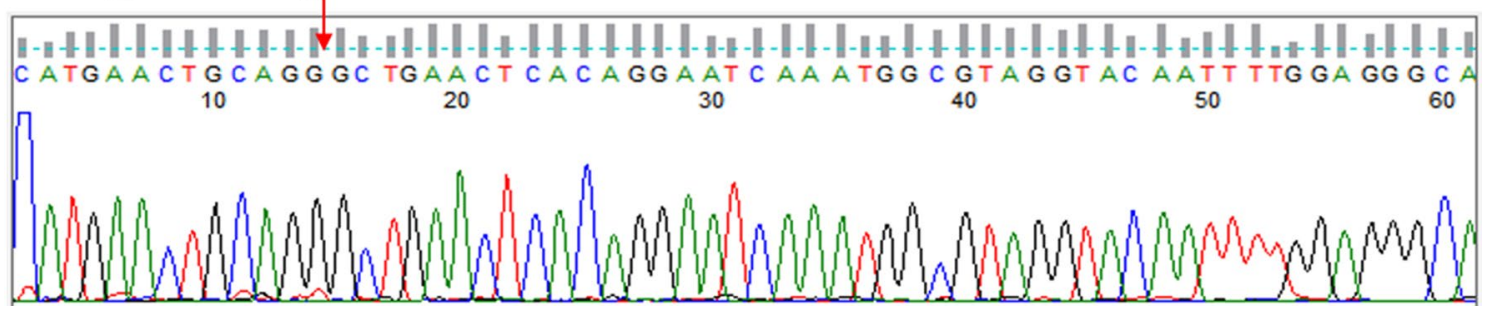

Fig. 3 continued

the above results suggested the different circRNA expression patterns in NAFLD livers, compared to the control group.

Additionally, GO terms and KEGG pathway were carried out to interpret the biological functions of 93 differentially expressed circRNAs. As shown in Fig. 4, the top 10 highly enriched GO biological process (BP) and 5 highly enriched GO cellular component (CC) and molecular function (MF) were demonstrated (Fig. 4a). The most enriched GO terms in BP was 'fatty acid metabolic process' $(p<0.0001)$, that in CC were 'endosome membrane' and 'endosome part', and that in MF were 'Ras GTPase binding' and 'small GTPase binding. KEGG pathway analysis was further conducted to find out the signalling pathway that the genes participate, which obtained eight significantly enriched pathways (Fig. 4b). According to the results of KEGG, 'cAMP signalling pathway' ranked the first among the differentially expressed circRNAs, which is considered to be associated with the attenuation of obesity in NAFLD.

\section{Validation of circRNA expression profiles by RT-qPCR}

To validate the sequencing results of circRNA expression profiles, we randomly selected eight differentially expressed circRNAs for RT-qPCR, including seven upregulated circRNAs and one downregulated circRNA. Primers for circRNA with forward and reverse sequences were illustrated in Fig. 3. The results of RT-qPCR suggested that both circRNA_0010514 and chr4_46734057_46749582 were upregulated, and these findings were the same as the results obtained from the RNA-seq data. Additionally, mmu_circ_0015959, mmu_circRNA_0010514, and chr7_82671604_82674582 reached the required expression level of statistical significance (Fig. 2e). On the other hand, the rest of the circRNAs had the opposite expression in RNA sequencing and RT-qPCR analysis, with no significant differences. The expected head-to-tail junctions of eight circRNAs was checked by Sanger sequencing (Fig. 3).

Moreover, a set of eight differentially expressed circRNAs were randomly selected for another round of 


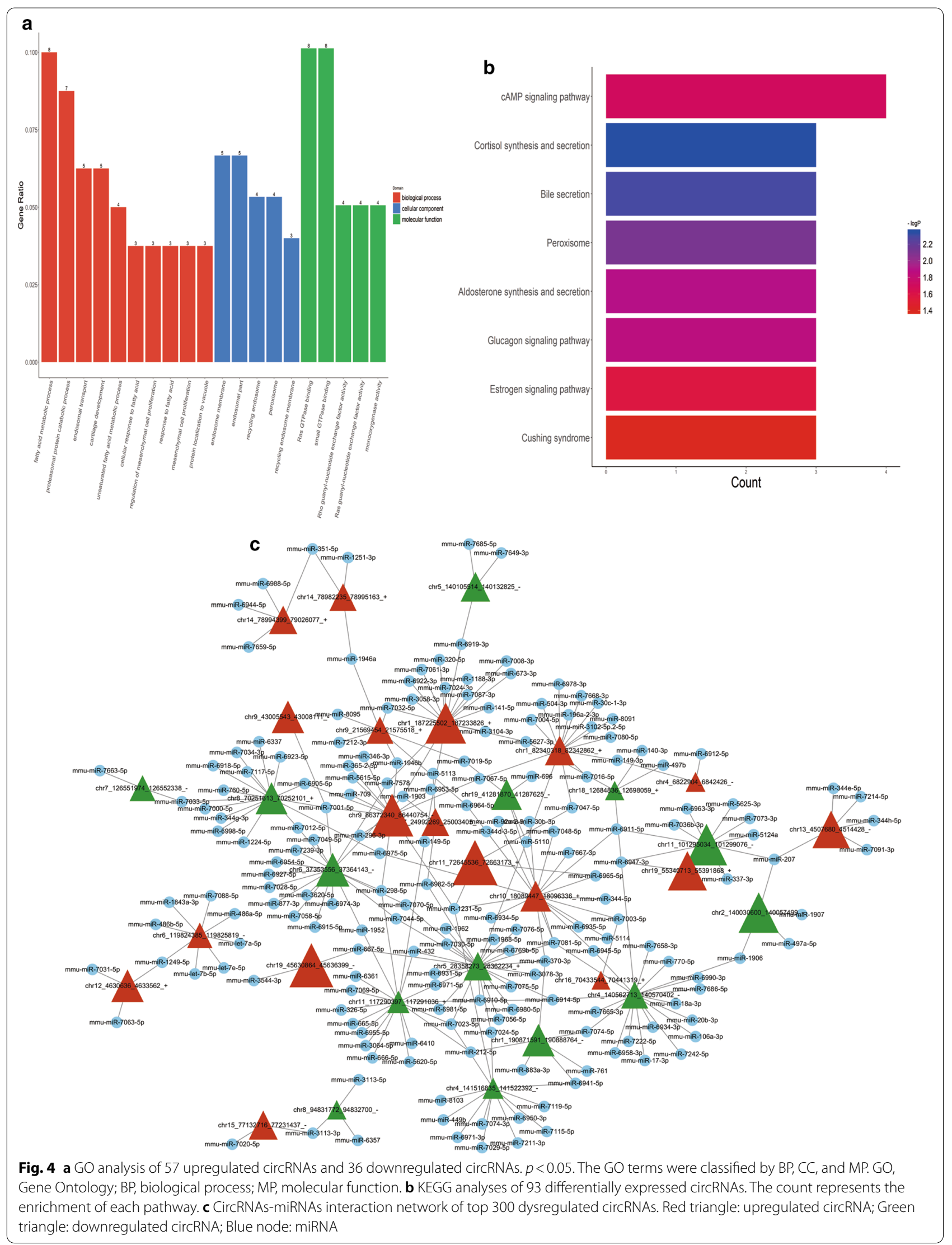


RT-qPCR validation, which reflected 6 out of 8 circRNAs had consistent results with the RNAseq data (Additional file 2: Fig. 1), among which the expression of chr1_82340318_82342862, chr6_37353556_37364143 and chr9_21742186_21742796 were statistically significant $(p<0.05)$. The primer sequence of each circRNA is present in Additional file 3: Table S2.

\section{Prediction of circRNA-miRNA network}

Given that circRNAs serve a significant biological role in the miRNA target, such as the regulation of gene expression. Herein, we selected the correspond miRNAs from miRbase database, and used miRanda software to detect the interaction between the miRNA with the differentially expressed circRNAs. The higher miRNA response elements (MRE) frequency, the more circRNAs binding sites with miRNA. We therefore built and displayed a circ-RNA-miRNA network of top 300 dysregulated circRNAs using software Cytoscape to exhibit the complex interaction (Fig. 4c and Additional file 4: Table S3).

\section{Discussion}

NAFLD is closely related with high-fat diet, obesity, insulin resistance, inflammation, and genetic factors [11]. Several studies have suggested that inflammation can contribute to the development of liver diseases, while Angulo [12] in 2002 has reported the close relationship of hepatic steatosis and obesity with NAFLD patients. A deeper understanding of the underlying mechanism of NAFLD is necessary for accurate diagnosis and new effective treatments. CircRNAs are found to be linked with many diseases, biological processes and gene expressions in earlier researches [13, 14]. Tissue-specific expression is one of the characteristics of circRNAs, which allows circRNA to inhibit the miRNAs activity, suggesting the regulatory role of circRNAs in the progression of diseases [4]. Previous studies have also indicated the close relationship between circRNAs with hepatic steatosis and NASH, and circRNAs can regulate the cancer cell growth, proliferation, migration and invasion. Guo et al. [3] have reported the dysregulation of circRNAs is related to the hepatic steatosis. To date, not many researchers have paid sufficient attention to the important role of circRNAs in NAFLD. Given the prevalence of NAFLD, further investigation of circRNAs profiles may provide insight into the pathogenic mechanism of NAFLD.

In our study, a long-term HFD-induced NAFLD in mouse model was firstly established, H\&E and ORO staining were applied to confirm the success of NAFLD model. The serum results combined with the staining analysis further confirmed a successful simulation of NAFLD in the mice (Fig. 1). After 32 weeks, the groups of NAFLD mice exhibited disordered hepatic lobules and fat accumulation in the liver, accompanied by significantly increased plasma TG, TC levels and liver weight $(p<0.05)$. The detected circRNAs of NAFLD mouse liver tissues were distributed on all the mouse chromosomes, however, not on the Y chromosome (Fig. 2a). In this work, 93 dysregulated circRNAs with the threshold of $\mathrm{FC} \geq 1.5$ and $\mathrm{p}<0.05$ were observed based on the heatmap and volcano plotting (Fig. 2b-d), including 57 upregulated and 36 downregulated circRNAs in the NAFLD group. Following validation by RT-qPCR, most of circRNAs were differentially expressed in NAFLD group. Moreover, compared to the control group, the significantly differentially expressed circRNAs were annotated in NAFLD group, and the circRNA-miRNA network was also predicted. Different circRNAs might have various potential miRNA targets. The target miRNAs of mmu_circRNA_0049392 are miR-7037-5p and miR-6919-5p, which have been validated as a receptor of low-density lipoprotein (LDL). A correlation analysis of mmu_circ_0049392 and miR-7037-5p with serum parameter was conducted and revealed a significant negative correlation between mmu_circ_0049392 and mmu_miR-7037-5p $(p<0.01, \quad|\mathrm{r}|=0.6977)$, suggesting that circRNA do function as 'miRNA sponge' to inhibit the activity of miRNAs, and therefore regulate the gene expression at transcription level (Fig. 5). The abnormal gene expression was found to be associated with atherosclerosis, obesity and diabetes. LDL receptor encoded by Ldlr gene allows the degradation of LDL [15]. A study conducted by Kelli et al., in 2017 [16] have reflected the attenuated disease development, decreased triglycerides accumulation and inflammation responses in $L d l r-/-$ mice fed with HFD. Hence, further experiments of the role of circRNA_0049392 in NAFLD is required.

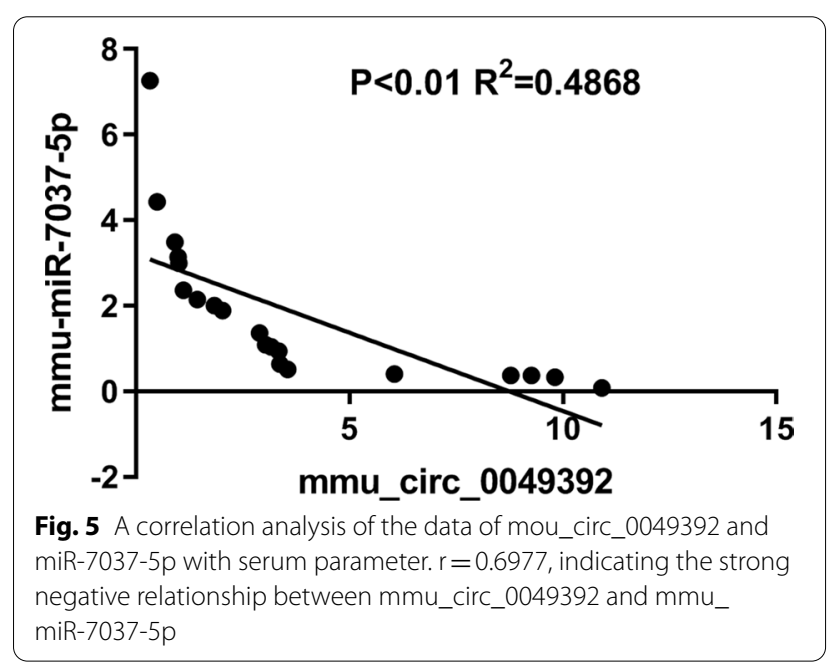



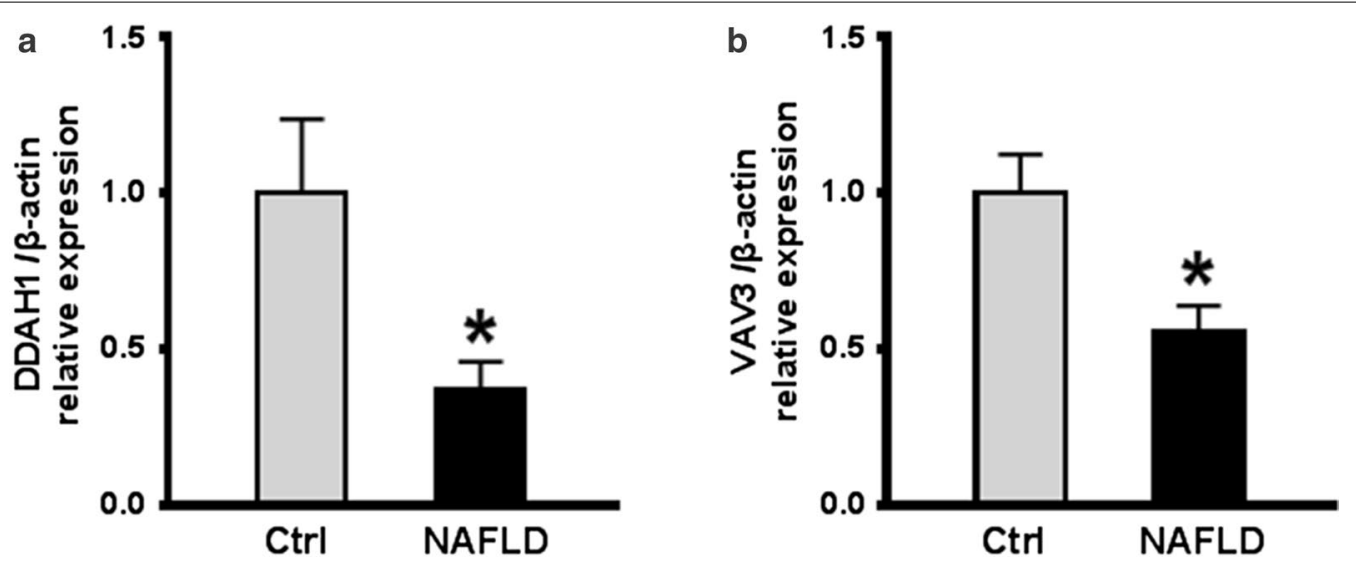

Fig. 6 The results of DDAH1 a and VAV3 $\mathbf{b}$ expression in control and NAFLD mice using RT-qPCR. ${ }^{*} p<0.05$

To explore the regulatory roles of cricRNAs in the pathogenesis of NAFLD, the GO terms of these genes in network were analyzed. We found that the circRNAs are enriched in the fatty acid metabolic process. In GO terms of MF and CC, these circRNAs were mostly enriched in small GTPase and endosome membrane, respectively. The results of KEGG pathway analysis indicated circRNAs were related to the signaling pathway of cAMP, and cAMP serves as a positive mediator in the attenuation of steatosis and obesity in NAFLD [17]. Additionally, $D D A H 1$ and $V A V 3$ genes were found to be associated with the development of NAFLD, while the target miRNA of circRNA chr3_145845704_145853276_+ and chr3_109562742_109578364_+ may be inhibited to regulate the DDAH1 and VAV3 expressions. Dimethylarginine Dimethylaminohydrolase 1 (DDAH1) is an enzyme that degrades Asymmetric dimethylarginine (ADMA), and is highly expressed in the liver [18], while increased ADMA levels are reported in NAFLD [19]. The ADMA-DDAH1 pathway was found to have a remarkable effect on the hepatic lipogenesis of HFD-induced mice, in other words, DDAH1 may protect against NAFLD via attenuating ADMA accumulation [18]. The decreased expression of DDAH1 in NAFLD mice indicated the protective effect of DDAH1 against NAFLD (Fig. 6a). Moreover, VAV3 is a Rho family GTPase guanine nucleotide exchange factor (GEF), and the activation of VAV3 in a pathway involves in the actin cytoskeletal rearrangement [20]. A previous study has also suggested the leukocytes often migrate and invade the hepatic lobules in the HFD-induced mice, while the VAV3-activated the pathway for leukocytes motility and direction sensing. The dropped VAV3 expression in model mice reflected that VAV3 played an important role in protecting mice from NAFLD (Fig. 6b). Taken together, the above findings suggested $D D A H 1$ and $V A V 3$ genes might serve as a potential biological marker of NAFLD development.

As shown in Fig. 7, a homologous sequence analysis of circ_0049392 comparing between mouse and human found that mouse shared nearly $89 \%$ of sequence homology with the sequence of has_circ_0049392 in human, however, the expression profile in human NAFLD still requires further investigation. In addition, it is not possible to exclude other dysregulated circRNAs that involved in the pathogenesis of NAFLD due to the low validation rate of RNAseq results with the RT-qPCR results, and therefore future study on this issue is required to provide a promising avenue for research. Moreover, it is not applicable to detect circRNA in human liver to confirm the early diagnostic biomarker of NAFLD.

\section{Conclusions}

In conclusion, we identified 93 dysregulated circRNAs in the NAFLD mouse liver tissues. We constructed a network to illustrate the association between differentially expressed circRNAs and their potential target miRNAs. $D D A H 1$ and $V A V 3$ genes were found to be associated with the development of NAFLD. This present study is the first one to explore the circRNAs profiles in NAFLD using RNA sequencing, RT-qPCR and bioinformatics analysis. This paper has demonstrated the significant role of circRNAs in the pathogenesis of NAFLD. 


\section{Sequence ID: Query_48885 Length: 259 Number of Matches: 1 \\ Range 1: 1 to 259 Graphics \\ $\nabla \underline{\text { Next Match }} \Delta \underline{P}$

\begin{tabular}{lllll}
\hline Score & Expect & Identities & Gaps & Strand \\
337 bits(373) & $3 e-97$ & $230 / 259(89 \%)$ & $0 / 259(0 \%)$ & Plus/Plus \\
\hline
\end{tabular} \\ Query 1 CTTCATGTACTGGACTGACTGGGGAACTCCCGCCAAGATCAAGAAAGGGGGCCTGAATGG 60

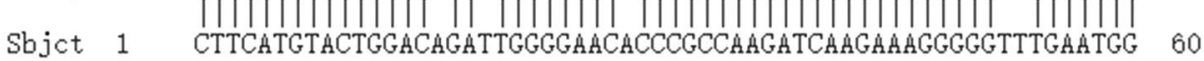 \\ Query 61 TGTGGACATCTACTCGCTGGTGACTGAAAACATTCAGTGGCCCAATGGCATCACCCTAGA 120

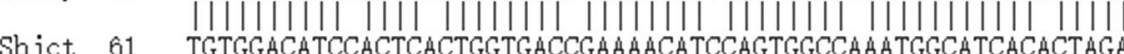 \\ Query 121 TCTCCTCAGTGGCCGCCTCTACTGGGTTGACTCCAAACTTCACTCCATCTCAAGCATCGA 180 \\ Sbjet 121 TCTTTCCAGTGGCCGTCTCTATTGGGTTGATTCCAAACTCCACTCTATCTCCAGCATCGA 180 \\ Query 181 TGTCAACGGGGGCAACCGGAAGACCATCTTGGAGGATGAAAAGAGGCTGGCCCACCCCTT 240 \\ sbjet 181 TGTCAATGGGGGCAATCGGAAAACCATTTTGGAGGATGAGAACCGGCTGGCCCACCCCTT 240 \\ Query 241 CTCCTTGGCCGTCTTTGAG 259 \\ Sbjet 241 CTCCTTGGCCATCTATGAG 259}

Fig. 7 The results of the homologous sequence analysis of circRNA_0049392 comparing between mouse (sbjct 1) and human (Query 1). The mouse shared $89 \%$ sequence homology compared with its sequence in humans. CircRNA, circular RNA

\section{Supplementary Information}

The online version contains supplementary material available at https://doi. org/10.1186/s12967-020-02637-w.

Additional file 1: Table S1. The fold-change and $p$ value of 93 differentially expressed circRNAs.

Additional file 2: Figure S1. The expression levels of another eight random selected circRNAs after RT-qPCR validation. ${ }^{*} p<0.05,{ }^{* *} p<0.01$.

Additional file 3: Table S2. Primers sequences of randomly selected eight circRNAs and two genes for another reverse transcription-quantitative polymerase chain-reaction (RT-qPCR)

Additional file 4: Table S3. The circRNAs-miRNAs interaction network of top 300 dysregulated circRNAs.

\section{Abbreviations}

NAFLD: Nonalcoholic fatty liver disease; circRNAs: Circular RNAs; HFD: High-fat diet; GO: Gene ontology; KEGG: Kyoto Encyclopedia of Genes and Genomes; NASH: Nonalcoholic steatohepatitis; HCC: Hepatocellular carcinoma; tRNA: Transfer RNA; miRNA: MicroRNA; GTT: Glucose tolerance test; ITT: Insulin tolerance test; TG: Triglyceride; TC: Total cholesterol; PBS: Phosphatebuffered saline; H\&E: Hematoxylin and eosin; ORO: Oil Red O; RT-qPCR: Reverse transcriptionquantitative polymerase chain-reaction; BP: Biological process; CC: Cellular components; MF: Molecular function; MRE: MiRNA response elements; LDL: Low-density lipoprotein; DDAH1: Dimethylarginine Dimethylaminohydrolase 1; ADMA: Asymmetric dimethylarginine; GEF: GTPase guanine nucleotide exchange factor.

\section{Acknowledgements}

Not applicable.

\section{Authors' contributions}

$X L Y, J J D, A Q D, S W, L G Z$ and $Y B P$ wrote, conceived and designed the experiments; XLY, LGZ and YBP collected the samples; XLY, JJD, AQD and SW analyzed and interpreted the data. All authors read and approved the final manuscript.

\section{Funding}

This work was supported by Shanghai Natural Science Foundation (19ZR1447500), Scientific Program of Shanghai Pudong Hospital (YJRCJJ201808, YJRCJJ201811, and YJRCJJ201903), Talents Training Program of Pudong Hospital affiliated to Fudan University (PJ202001 and PY202002) and Science and Technology Development Fund of Shanghai Pudong New Area (PKJ2019-Y37).

\section{Availability of data and materials}

The datasets during and/or analyzed during the current study available from the corresponding author on reasonable request.

\section{Ethics approval and consent to participate}

This study was approved by the Animal Research Ethical Committee of the Fudan University Pudong Medical Center. All experimental procedures followed the Guidelines for the Care and Use of Laboratory Animals of the National Institute of Health in China.

\section{Consent for Publication}

Not applicable.

\section{Competing interests}

The authors declare that they have no competing interests.

\section{Author details}

${ }^{1}$ Department of Endocrinology and Metabolic Diseases, Shanghai Pudong Hospital, Fudan University Pudong Medical Center, 2800 Gongwei Road, Pudong, Shanghai 201399, China. ${ }^{2}$ Department of Emergency, Shanghai Pudong Hospital, Fudan University Pudong Medical Center, 2800 Gongwei Road, Pudong, Shanghai 201399, China. ${ }^{3}$ Department of Stomatology, Shanghai Pudong Hospital, Fudan University Pudong Medical Center, 2800 Gongwei Road, Pudong, Shanghai 201399, China. ${ }^{4}$ Department of Nephrology, Shanghai Pudong Hospital, Fudan University Pudong Medical Center, 2800 Gongwei Road, Pudong, Shanghai 201399, China.

Received: 13 Auqust 2020 Accepted: 27 November 2020

Published online: 11 December 2020 


\section{References}

1. Qu Y, Deng C, Luo Q, Shang X, Wu J, Shi Y, et al. Arid1 la regulates insulin sensitivity and lipid metabolism. EBioMedicine. 2019;42:481-93.

2. Guo X, Sun F, Chen J, Wang Y, Pan Q, Fan J. circRNA_0046366 inhibits hepatocellular steatosis by normalization of PPAR signaling. World J Gastroenterol. 2018;24(3):323-37.

3. Guo X, He C, Wang Y, Sun C, Li G, Su Q, et al. Circular RNA profiling and bioinformatic modeling identify its regulatory role in hepatic steatosis. Biomed Res Int. 2017:2017:5936171.

4. Xu T, Wu J, Han P, Zhao Z, Song X. Circular RNA expression profiles and features in human tissues: a study using RNA-seq data. BMC Genomics. 2017;18(Suppl 6):680.

5. Chen Y, Huang H, Xu C, Yu C, Li Y. Long non-coding RNA profiling in a non-alcoholic fatty liver disease rodent model: new insight into pathogenesis. Int J Mol Sci. 2017. https://doi.org/10.3390/ijms18010021.

6. Jin X, Feng C, Xiang Z, Chen Y, Li Y. CircRNA expression pattern and circRNA-miRNA-mRNA network in the pathogenesis of nonalcoholic steatohepatitis. Oncotarget. 2016;7(41):66455-67.

7. Sulaiman SA, Muhsin NIA, Jamal R. Regulatory non-coding RNAs network in non-alcoholic fatty liver disease. FrontPhysiol. 2019. https://doi. org/10.3389/fphys.2019.00279.

8. Fan X, Zhang X, Wu X, Guo H, Hu Y, Tang F, et al. Single-cell RNA-seq transcriptome analysis of linear and circular RNAs in mouse preimplantation embryos. Genome Biol. 2015;16(1):148.

9. Wang Y, Zhang H, Wang J, Li B, Wang X. Circular RNA expression profile of lung squamous cell carcinoma: identification of potential biomarkers and therapeutic targets. Biosci Rep. 2020. https://doi.org/10.1042/BSR20 194512.

10. Ou Q, Zhao Y, Zhou J, Wu X. Comprehensive circular RNA expression profiles in a mouse model of nonalcoholic steatohepatitis. Mol Med Rep. 2019:19(4):2636-48.

11. Gaggini M, Carli F, Rosso C, Buzzigoli E, Marietti M, Latta VD, et al. Altered amino acid concentrations in NAFLD: Impact of obesity and insulin resistance. Hepatology. 2017;67(1):145-58.

12. Angulo P. Nonalcoholic fatty liver disease. The New England journal of medicine. 2002;346(16):1221-31.
13. Tian C, Tang X, Zhu X, Zhou Q, Guo Y, Zhao R, et al. Expression profiles of circRNAs and the potential diagnostic value of serum circMARK3 in human acute Stanford type A aortic dissection. PLoS ONE. 2019;14(6):e0219013.

14. Shen Y, Pan J, Sun Z, Chen X, Zhou X, Zhou X, et al. Differential expression of circRNAs during rat lung development. Int J Mol Med. 2019:44(4):1399-413.

15. Mbikay M, Mayne J, Sirois F, Fedoryak O, Raymond A, Noad J, et al. Mice fed a high-cholesterol diet supplemented with quercetin-3-glucoside show attenuated hyperlipidemia and hyperinsulinemia associated with differential regulation of PCSK9 and LDLR in their liver and pancreas. Mol Nutr Food Res. 2018;62(9):e1700729.

16. Lytle KA, Wong CP, Jump DB. Docosahexaenoic acid blocks progression of western diet-induced nonalcoholic steatohepatitis in obese Ldlr-/- mice. PLOS ONE. 2017;12(4):e0173376.

17. Wahlang B, McClain C, Barve S, Gobejishvili L. Role of CAMP and phosphodiesterase signaling in liver health and disease. Cell Signal. 2018;49:105-15.

18. Li T, Feng R, Zhao C, Wang Y, Wang J, Liu S, et al. Dimethylarginine dimethylaminohydrolase 1 protects against high fat diet induced hepatic steatosis and insulin resistance in mice. Antioxid Redox Signal. 2017;26(11):598-609.

19. Boga S, Alkim H, Koksal AR, Bayram M, Ozguven MBY, Ergun M, et al. Increased plasma levels of asymmetric dimethylarginine in nonalcoholic fatty liver disease: relation with insulin resistance, inflammation, and liver histology. J Investig Med. 2015;63(7):871-7.

20. Xia J, Yuan J, Xin L, Zhang Y, Kong S, Chen Y, et al. Transcriptome analysis on the inflammatory cell infiltration of nonalcoholic steatohepatitis in Bama Minipigs induced by a long-term high-fat, high-sucrose diet. PLoS ONE. 2014;9(11):e113724.

\section{Publisher's Note}

Springer Nature remains neutral with regard to jurisdictional claims in published maps and institutional affiliations.
Ready to submit your research? Choose BMC and benefit from:

- fast, convenient online submission

- thorough peer review by experienced researchers in your field

- rapid publication on acceptance

- support for research data, including large and complex data types

- gold Open Access which fosters wider collaboration and increased citations

- maximum visibility for your research: over $100 \mathrm{M}$ website views per year

At $\mathrm{BMC}$, research is always in progress.

Learn more biomedcentral.com/submissions 